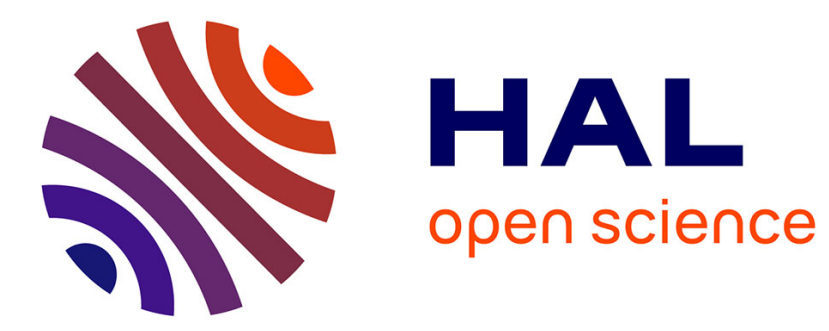

\title{
Draw Ratio at the Onset of Strain-Induced Crystallization in Cross-Linked Natural Rubber \\ Pierre-Antoine Albouy, Paul Sotta
}

\section{To cite this version:}

Pierre-Antoine Albouy, Paul Sotta. Draw Ratio at the Onset of Strain-Induced Crystallization in Cross-Linked Natural Rubber. Macromolecules, 2020, 53 (3), pp.992-1000. 10.1021/acs.macromol.9b01957 . hal-03016574

\section{HAL Id: hal-03016574 https://hal.science/hal-03016574}

Submitted on 23 Nov 2020

HAL is a multi-disciplinary open access archive for the deposit and dissemination of scientific research documents, whether they are published or not. The documents may come from teaching and research institutions in France or abroad, or from public or private research centers.
L'archive ouverte pluridisciplinaire HAL, est destinée au dépôt et à la diffusion de documents scientifiques de niveau recherche, publiés ou non, émanant des établissements d'enseignement et de recherche français ou étrangers, des laboratoires publics ou privés. 


\title{
DRAW RATIO AT ONSET OF STRAIN-INDUCED CRYSTALLIZATION IN CROSS- LINKED NATURAL RUBBER.
}

\author{
*Pierre-Antoine Albouy ${ }^{\mathrm{a}}$ and Paul Sotta ${ }^{\mathrm{b}}$
}

${ }^{a}$ Laboratoire de Physique des Solides, UMR 8502, Université Paris-Sud, Université ParisSaclay, 91405 Orsay, France

${ }^{b}$ Laboratoire Polymères et Matériaux Avancés, UMR 5268, CNRS/ Solvay, R\&I Centre Lyon, 69192 Saint-Fons, France

Corresponding Author: E-mail pierre-antoine.albouy@u-psud.fr; Tel.: +33-169156049; fax: +33-169156086

Keywords: Natural Rubber, Strain-induced Crystallization, Crystallization kinetics

\begin{abstract}
The first theory of strain-induced crystallization in regular elastomers was laid out some seventy years ago by Flory. He identified the driving mechanism that concurs to the stabilization of the crystalline phase under stretching as the relaxation of the remaining amorphous phase due to the growth of oriented crystallites. However Flory's theory is an equilibrium theory and does not apply to dynamic conditions that are kinetically controlled. We presently performed careful measurements of the crystallinity index and draw ratio for the amorphous phase during mechanical cycling and step-wise extension so as to enlighten their intricate interplay. We propose a simple description of the crystallization process that allows establish a quantitative relation between these two parameters and predict their timeevolution. This analysis leads to the definition of a master curve for the time-variation of the crystallinity index.
\end{abstract}




\section{Introduction}

More than ninety years after its discovery by Katz in natural rubber (NR), strain-induced crystallization (SIC) remains the subject of intense research ${ }^{1}$. Since then this fascinating effect has been observed in many elastomers including polychloroprene rubber (CR), cis- and trans- polybutadiene, polyisobutylene or thermoplastic polyurethanes ${ }^{2,3,4,5}$. It is known to improve the mechanical and ultimate properties of the material, although the effective mechanisms at play are still a matter of debate. The basic driving force for SIC has been clearly established by Flory and consist in (i) a lowering of the entropy cost for the incorporation of pre-stretched chain segments into crystallites, (ii) entropy release into the amorphous fraction accompanying crystallite growth ${ }^{6}$. Although the general framework of Flory's theory relies on thermodynamic equilibrium, this driving force remains effective in out-of-equilibrium conditions that are most often encountered in practical cases. Along these lines interesting models have been developed to account for the evolution of the crystalline content and retraction force during cyclic deformation, but, apart their relative complexity, they often resort to Flory's equilibrium formula ${ }^{7}$.

X-ray diffraction is a preferred method to investigate SIC and it was undoubtedly boosted during the last twenty years by an easier access of research teams to synchrotron facilities ${ }^{8}$. However most published X-ray studies of SIC in elastomers concentrate on quantifying the crystalline content or the orientation and dimensions of crystallites, but somehow neglect the structural changes that occur into the amorphous fraction and in particular the evolution of its strain state. The link between the crystalline content and the strain in the amorphous fraction 
is central to Flory's theory and has been clearly demonstrated both in equilibrium and in outof-equilibrium conditions. Indeed, we have recently shown that the crystallite growth in NR and CR is accompanied by a considerable limitation of the actual strain (or equivalently draw ratio $\lambda_{A m}$ during stretching) in the remaining amorphous fraction ${ }^{9,10}$. More precisely, beyond SIC onset, $\lambda_{A m}$ becomes significantly lower than the applied draw ratio $\lambda$. While it still continues to increase in $\mathrm{CR}$, a nearly flat plateau is observed in NR, with $\lambda_{A m}$ remaining close to the draw ratio $\lambda_{\text {Onset }}$ at crystallization onset. We coined this last spectacular effect a "strainregulation process" to insist upon the active role played by crystallization. This effect could actually constitute a protective mechanism against tear.

Thus the macroscopic draw ratio $\lambda_{\text {Onset }}$ emerges as a key microscopic parameter characterizing the amorphous fraction in a strained-crystallized elastomer. This strongly suggests that this parameter conveys a deep physical significance. In fact, considering the draw ratio of the amorphous phase may be much more informative than considering the macroscopically measured stress, as this latter quantity is subjected to complex selfreinforcement effects in the semi-crystalline material.

SIC kinetics in NR and CR has been also assessed by various authors using so-called tensile impact or strain jump tests where the sample is submitted to stepwise loading ${ }^{10,11,12}$. $\lambda_{\text {Onset }}$ is generally not considered explicitly in this type of measurements. One motivation for the present study is to investigate its potential implicit role, or, stated in another way, to elaborate a kinetic definition of $\lambda_{\text {Onset }}$.

The paper is organized as follows. The experimental section includes the description of the material and a short description of the X-ray set-up and data treatment that have been already detailed elsewhere. The next section is devoted to cyclic elongation tests at various drawing speeds and temperatures: its primary goal is to obtain an extensive, quantitative knowledge of $\lambda_{\text {Onset }}$ for these samples, necessary for the subsequent discussion of tensile impact tests. It 
offers also the opportunity of a deeper discussion of the mechanism of strain regulation. A simple semi-quantitative relation is furthermore proposed between the amount of strain relaxation for the amorphous phase and the crystallinity index. Tensile impact tests are presented in the last section with a particular attention to the evolution of $\lambda_{A m}$ during the crystallization process: the results suggest a new interpretation for $\lambda_{\text {Onset }}$ and this offers a simplified and comprehensive description of SIC in out-of-equilibrium conditions.

\section{Experimental section}

\subsection{Materials.}

The material is a natural rubber of grade SMR 5L vulcanized by sulfur ( $1.5 \mathrm{phr})$ in the presence of conventional activators and antioxidant agents (see detailed formulation in Table 1). The average molecular weight between cross-links is $6330 \mathrm{~g} \cdot \mathrm{mol}^{-1}$ (86 isoprene units), slightly less than the admitted value for the average molecular weight between entanglements in NR, ca. 7000 g.mol ${ }^{-1}$. The ratio $C_{2} / C_{l}$ for the Mooney-Rivlin constants is 0.31 . It is recalled that sulfur vulcanization leads to a quite homogeneous cross-link density according to proton multiple quantum NMR ${ }^{13}$. The test samples have a dumbbell shape and are about $20 \mathrm{~mm}$ long, $1.4 \mathrm{~mm}$ thick and ca.7 $\mathrm{mm}$ wide and they are systematically pre-cycled three times up to a maximum elongation $\lambda=6$ before use. Each data set presented here is an average over three test samples.

\begin{tabular}{|c|c|}
\hline Component & Amount (phr) \\
\hline Natural Rubber SMR 5L & 100 \\
\hline Sulfur & 1.5 \\
\hline CBS & 2 \\
\hline Zinc oxide & 4 \\
\hline
\end{tabular}




\begin{tabular}{|c|c|}
\hline Stearic acid & 2 \\
\hline 6PPD & 1.9 \\
\hline TBzTD & 0.2 \\
\hline
\end{tabular}

Table 1. Formulation of vulcanized rubber sample. CBS: N-cyclohexyl-2-benzothiaxyl (primary accelerator); 6PPD: N-(1,3-dimethylbutyl)-N'-phenyl-P-phenylenediamine (oxidation inhibitor); TBzTD: tetrabenzyl thiuram disulfide (secondary accelerator).

\subsection{Stretching apparatus.}

The set-up allows acquiring X-ray patterns during mechanical cycling at various constant drawing/retraction speeds ( 1 to $400 \mathrm{~mm} / \mathrm{min}$, corresponding to strain rates of $8.33 \times 10^{-4}$ to $\left.3.33 \times 10^{-1} \mathrm{~s}^{-1}\right)$ and various temperatures $\left(0^{\circ} \mathrm{C}\right.$ to $\left.100^{\circ} \mathrm{C}\right)$. For tensile impact tests, two pneumatic springs can be attached onto the two opposite moving parts and the transient step duration is ca. $10 \mathrm{~ms}^{9}$. In all cases, temperature regulation is obtained by blowing thermalized air onto the whole sample surface, which helps limit temperature changes occurring during rapid crystallization or melting processes. The apparatus is mounted on a rotating anode generator operated at $40 \mathrm{kV}, 40 \mathrm{~mA}$ and the $\mathrm{CuK}_{\alpha}$ radiation emitted by the copper target is isolated by a doubly-curved graphite monochromator. A laboratory X-ray generator has the advantage of availability and absence of radiation damages compared to synchrotron radiation. Furthermore the sensitivity of modern hybrid pixel X-ray cameras partially compensate for the lower flux: as an example, patterns collected with the shorter and longer acquisition durations selected for this study are given in Figure 1. Acquisition times as low as $0.1 \mathrm{~s}$ are possible but do not offer a significantly increased time resolution as the detector transfer time between each acquisition is ca. $0.3 \mathrm{~s}$. 


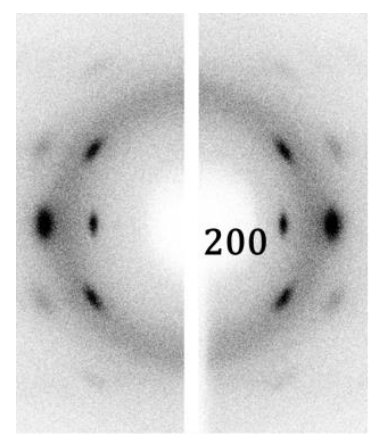

$0.4 \mathrm{~s}$

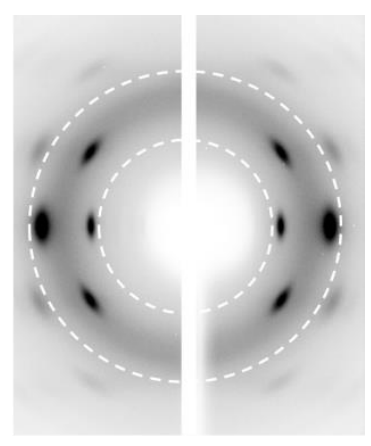

$20 \mathrm{~s}$

Figure 1. Diffraction patterns of a partially crystallized sample $(\lambda \approx 5.3)$. Initial strain rate: $8.310^{-2} \mathrm{~s}^{-1}$ (left) and $8.310^{-4} \mathrm{~s}^{-1}$ (right) (rotating anode generator $40 \mathrm{kV}, 40 \mathrm{~mA}$. Hybrid pixel camera; the blind vertical zone corresponds to the gap between the two detecting elements).

\subsection{Data processing.}

Relevant data are retrieved from angular scans centered on the amorphous halo, where a significant fraction of the diffracted intensity is concentrated (the region in between the white dashed circles in Figure 1-right). The procedure used to separate the respective contributions of the crystalline and amorphous phases is briefly recalled ${ }^{9}$. A crystallinity index (denoted $C$ ) is presently defined within this limited zone as the ratio of the integrated crystalline intensity to the total scattered intensity corrected for the air scattering contribution (Compton contribution is negligible). It is indicative for the absolute crystalline content (denoted $\chi$ in what follows) that needs to be evaluated over the whole scattering space. The azimuthal profiles of the crystalline peaks are adjusted by Pearson VII functions, of the form $(1+$ $\left.\left(\varphi-\varphi_{0}\right) / \tau\right)^{-\mu}$, where $\varphi$ is the azimuth angle. The actual $\mu$-value is fixed by adjusting some azimuthal peak profiles for the 200 reflection (see Figure 1-left) after careful correction for amorphous background. It is found to be close to 5 for mechanical cycling and to 6 for tensile tests. Such high $\mu$-values point to nearly Gaussian profiles and their difference only reflects 
minute changes in the orientation distribution function for crystallites. Uniaxial stretching induces a modulation of the amorphous intensity that remains weak and can be adjusted by a function $A+B \cos ^{2} \varphi$. The associated Legendre coefficient $\left\langle P_{2}^{R X}\right\rangle=\left(3\left\langle\cos ^{2} \varphi\right\rangle+1\right) / 2$ is assumed to be proportional to the Herman coefficient $\left\langle P_{2}\right\rangle$ for the segmental orientation function of the molten polymer chains. It is recalled that $\left\langle P_{2}\right\rangle=\left(\lambda^{2}-1 / \lambda\right) / 5 N$ within the framework of the Gaussian theory of rubber elasticity, where $\lambda$ is the draw ratio and $N$ is the average number of freely jointed statistical segments per chain ${ }^{14}$.

\section{Experimental results}

\subsection{Cyclic elongation}

\subsubsection{Relationship between $\left\langle P_{2}^{R X}\right\rangle$ and $\lambda_{A m}$.}

It has been clearly established in CR and NR-based compounds that, in the absence of crystallization, the measured quantity $\left\langle P_{2}^{R X}\right\rangle$ is proportional to $\lambda^{2}-1 / \lambda$ and that the proportionality factor is independent of temperature and proportional to the cross-link density, in full agreement with the theory of rubber elasticity quoted above [15]. For the present compound, the relationship

$$
\left\langle P_{2}^{R X}\right\rangle \approx-0.0014 \cdot\left(\lambda^{2}-1 / \lambda\right)
$$

holds during stretching and in the absence of SIC for all investigated drawing speeds and temperatures, as shown by the red lines in Figures 2-A and B. The negative sign in formula (1) simply originates from the fact that essentially inter-chain positional correlations are at the origin of the amorphous halo, which gives enhanced scattering perpendicular to the tensile direction. That $\left\langle P_{2}^{R X}\right\rangle$ does not depend on the drawing rate indicates that the relaxation time of the elastomer matrix is much faster than the time scale set by the fastest investigated drawing speed at the considered temperature. This gives rise to an effectively elastic-like behavior for 
chain segment orientation, without measurable dissipative-like (i.e. strain-rate dependent) contribution.

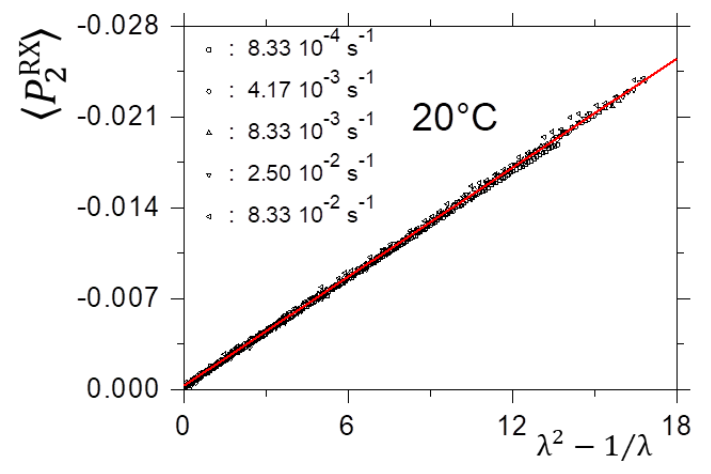

$-\mathbf{A}-$

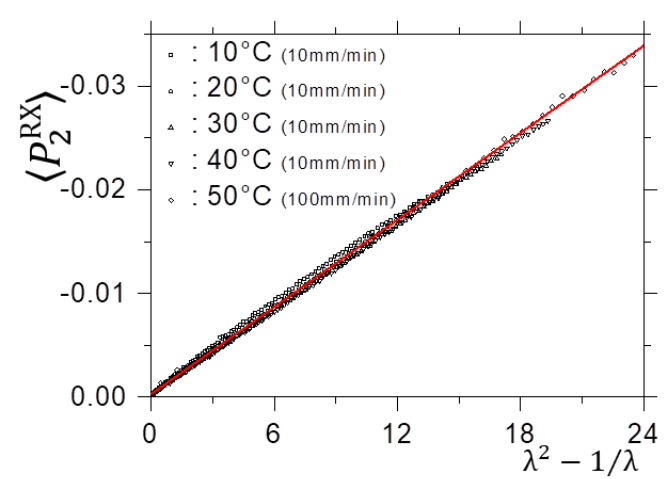

-B-

Figure 2. The second-order Legendre coefficient $\left\langle P_{2}^{R X}\right\rangle$ during stretching as a function of the elongation parameter $\lambda^{2}-1 / \lambda$ where $\lambda$ is the draw ratio, for different strain rates (A) and temperatures (B).

Once the sample partly crystallizes, equation (1) must be modified using the draw ratio of the amorphous fraction $\lambda_{A m}$ instead of the applied, macroscopic draw ratio $\lambda$ and taking into account the shortening of the active amorphous chains expected from crystallite growth. Along these lines we recently proposed to correct the average number of segment per chain $N$ in formula (1) by a factor $1-C^{16}$. However the impact of this correction is limited and its foundation is uncertain. In the present case, the crystallinity index remains low and for the sake of simplicity we retain the simplified formula

$$
\left\langle P_{2}^{R X}\right\rangle \approx-0.0014 .\left(\lambda_{A m}^{2}-1 / \lambda_{A m}\right)
$$

\subsubsection{Definition of characteristic draw ratios.}

Figure 3 is representative of the dependence of the crystallinity index $C$ upon elongation. Relevant draw ratio values are indicated. Only the intermediate draw ratio $\lambda_{\text {Int }}$ where $C$ 
variation presents a change in slope during recovery is unambiguously defined. To define the elongations at crystallization onset and melting, an intuitive way is to refer to a detection threshold for crystalline content. In the present case, the threshold at which crystallization is unambiguously detected by visual inspection of diffraction patterns corresponds to a crystallinity index of ca. 0.002 . The corresponding draw ratios in traction and recovery are reported in Figure 3 as "detection limit". However this definition is not intrinsic as it depends on the experimental sensitivity, and we prefer to set $\lambda_{\text {Onset }}$ and $\lambda_{\text {Melt }}$ by linear extrapolation (see Figure 3). The elongation values defined in this way actually coincide with the limits where the impact of SIC on $\left\langle P_{2}^{R X}\right\rangle$ or on the engineering stress becomes effective. These effects are briefly recalled below.

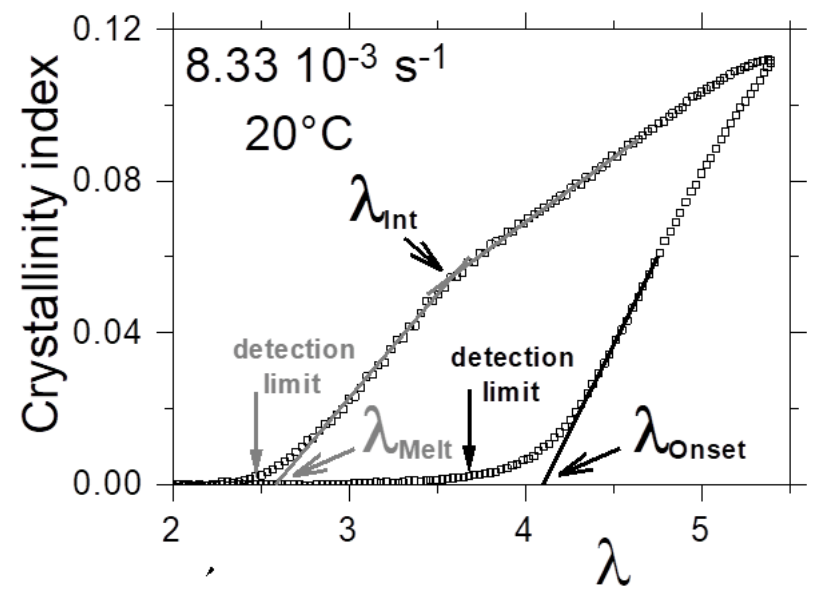

Figure 3. Representative crystallinity index $C$ versus draw ratio $\lambda$ diagram obtained during cyclic deformation.

The first effect of SIC during stretching is a slight stress-softening observed shortly after $\lambda_{\text {Onset }}$ is reached, well-above the detection limit (see Figure 4-A). This corresponds as well to the point where $\left\langle P_{2}^{R X}\right\rangle$ starts to level off (see Figure 4-B). This effect is attributed to SICinduced strain relaxation. Stress-hardening starts at higher $C$ values, while $\left\langle P_{2}^{R X}\right\rangle$ remains nearly constant. This last observation somehow contradicts the hypothesis that stress- 
hardening could be explained by an increasing effective physical cross-linking of the amorphous phase due to SIC. To explain stress-hardening, we rather favor the formation of a reinforcing crystallite network percolating through the sample ${ }^{9}$. At the starting point of retraction, the engineering stress drops rapidly, possibly as a result of the disintegration of the crystallite network, while $\left\langle P_{2}^{R X}\right\rangle$ decreases with a slope nearly equal to the slope of the upward branch of the cycle. $\lambda_{\text {Int }}$ approximately coincides with the onset of a plateau for both the stress and $\left\langle P_{2}^{R X}\right\rangle$. Its physical interpretation is discussed below. Below $\lambda_{\text {Melt }}$ (or more precisely below some characteristic value within the interval $\lambda_{\text {Melt }}$ - detection limit), there is a tiny difference between the upward and downward branches in both diagrams A and B, which can be attributed to reversible, physical creep solely (disentanglement or retraction of dangling chains), as the sample is fully amorphous in this regime. This small difference in both parts of the cycles could be adjusted with an apparent drop by ca. $8 \%$ of the initial crosslink density. However, the initial behavior is totally recovered by mild annealing. The engineering stress expected in the absence of SIC (grey curve in Figure 4-A) was obtained at $60^{\circ} \mathrm{C}$ (temperature at which SIC onset is beyond $\lambda=5$ ) and corrected by a temperature factor such that curves overlap below SIC onset .

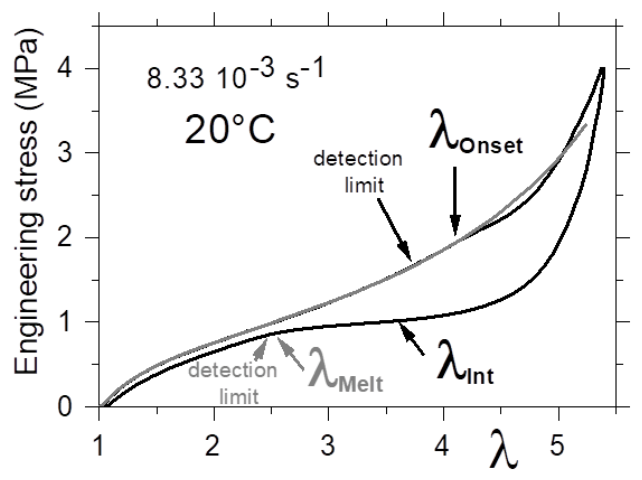

$-A-$

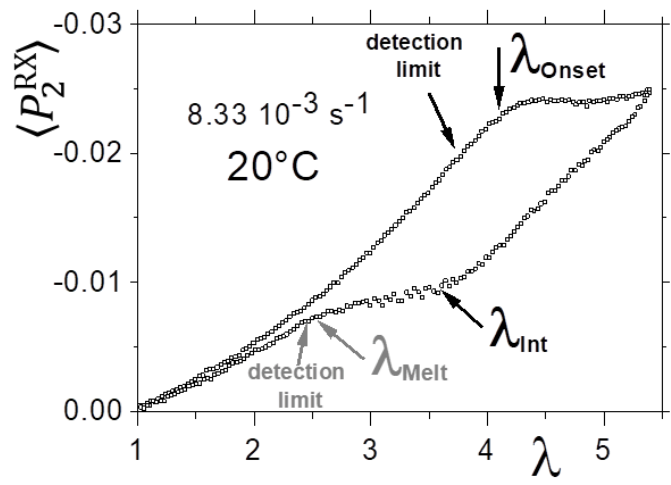

-B- 
Figure 4. Representative diagrams of the engineering stress (A) and $\left\langle P_{2}^{R X}\right\rangle$ parameter (B) versus elongation obtained during cyclic deformation. The grey line in A corresponds to the curve expected in the absence of SIC.

\subsubsection{Strain regulation effect.}

By applying equation (2) a value $\lambda_{A m}$ can be associated to each value of $\left\langle P_{2}^{R X}\right\rangle$ and a $\lambda_{A m}$ vs $\lambda$ diagram, similar to Figure 4-B, can be obtained. This is plotted in Figure 5-A. This graph offers an interesting perspective on the physical effects of SIC. During stretching, $\lambda_{A m}$ remains equal to the applied, macroscopic draw ratio until $\lambda_{\text {Onset }}$ is reached $(C \approx 0.01)$. Beyond $\lambda_{\text {Onset }}$, $\lambda_{A m}$ remains nearly constant. This is the so-called "strain-regulation" regime, visualized by a horizontal grey segment in Figure 5-A.

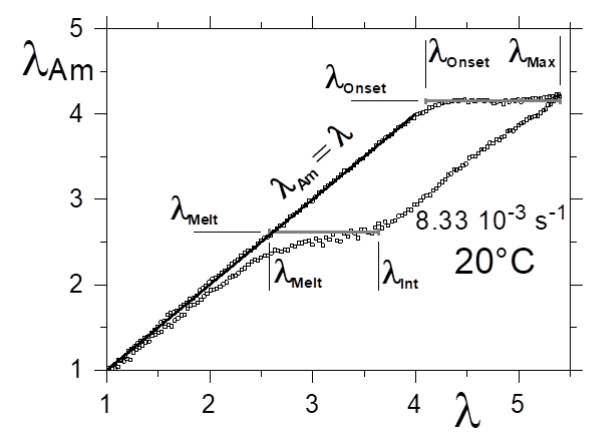

Figure 5. - The local computed elongation of the amorphous fraction $\lambda_{A m}$ as a function of the macroscopic elongation $\lambda$

This plateau corresponds to a kinetic equilibrium driven by the drawing velocity and the rate of strain relaxation within the amorphous phase related to the crystallization kinetics. The amount of relaxation $\lambda-\lambda_{A m}$ in relation to the absolute crystalline content (denoted $\chi$ ) can be evaluated following Flory's original calculation. We recently proposed a slightly different 
approach based on an analogy with the liquid-gas transition and the application of the lever rule ${ }^{16}$.

$$
\chi \approx \frac{\lambda-\lambda_{A m}}{\lambda_{X}-\lambda_{A m}}
$$

Where $\lambda_{A m}$ is the draw ratio of the amorphous phase and $\lambda_{X}$ the draw ratio of the crystalline phase. These parameters should be considered as thermodynamic parameters describing each phase ${ }^{16} \cdot \lambda_{X}$ is in fact the main adjustable parameter in the fitting of experimental data. The draw ratio in the crystalline phase may be identified to the maximum draw ratio of fully extended chains and thus must be of order $\lambda_{X}=\sqrt{3 N}^{16}$. Equation (3) can be rewritten as

$$
\lambda_{A m}=\lambda-\chi\left(\lambda_{X}-\lambda_{A m}\right)
$$

By taking the time derivative of Eq. (4), considering that both $\chi$ and $\lambda_{A m}$ depend on time, the rate of change of $\lambda_{A m}$ is thus given by

$$
(1-\chi) \frac{d \lambda_{A m}}{d t}=\frac{d \lambda}{d t}-\frac{d \chi}{d t}\left(\lambda_{X}-\lambda_{A m}\right)
$$

For simplicity we may neglect the variation of the correction prefactor $(1-\chi)$ and keep it equal to one, which does not change the overall argument. The strain-regulation effect corresponding to $\lambda_{A m}=c s t$, i.e. to $d \lambda_{A m} / d t=0$, thus corresponds to a regime in which the crystallization rate $d \chi / d t$ is locked to the drawing rate $d \lambda / d t$.

We make the hypothesis that the crystallization rate is controlled by the nucleation rate only and this point is discussed later. According to classical nucleation theory, the crystallization rate is given by

$$
\frac{d \chi}{d t}=v_{0} \exp \left[-\frac{\Delta H\left(\lambda_{A m}\right)}{k_{B} T}\right]
$$


Where $v_{0}$ is a nucleation attempt frequency and $\Delta H\left(\lambda_{A m}\right)$ is the nucleation barrier that is insomuch lowered as $\lambda_{A m}$ is higher than $\lambda_{\text {melt }}$ (the drawing ratio corresponding to equilibrium melting at the considered temperature). For a given, imposed drawing velocity $/ d t, \lambda_{\text {onset }}$ should then be effectively given by the condition

$$
\frac{d \lambda}{d t}=v_{0} \exp \left[-\frac{\Delta H\left(\lambda_{\text {onset }}\right)}{k_{B} T}\right]\left(\lambda_{X}-\lambda_{\text {onset }}\right)
$$

This kinetic equilibrium is schematized in Fig 6. This 'strain-regulation effect' is evidenced experimentally both by the plateau in $\lambda_{A m}$ in Figure 5-A (or equivalently Figure 4-B) and by the experimental observation that the crystallization rate $d \chi / d \lambda$ is nearly constant (for a fixed strain rate) along the stretching branch of the cycle beyond $\lambda_{\text {onset }}$ as shown in Figure 3 . According to equation (6), this means that the crystallization rate $v_{0} \exp \left[\Delta H\left(\lambda_{A m}\right) / k_{B} T\right]$ and hence $\lambda_{A m}$ remain nearly constant for a constant draw rate $d \lambda / d t$.

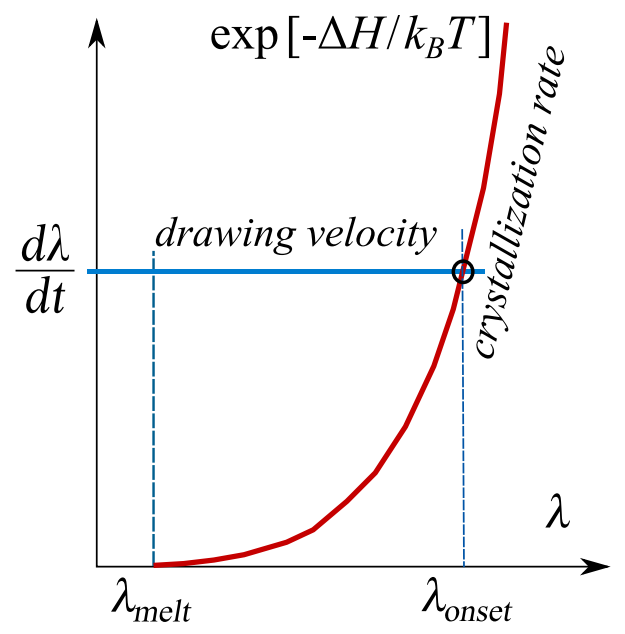

Figure 6. Schematics illustrating the strain-regulation effect. The crystallization rate is displayed on vertical axis, $\lambda_{\text {onset }}$ is determined by Eq. (7) relating the drawing rate to crystallization rate.

The validity of Equation (3) that relates $\chi$ and strain-relaxation has been tested based on the $\lambda_{A m}$ curve measured on both stretching and recovery branches of the cycle. For this 
purpose it is assumed that the measured crystallinity index $C$ as defined in paragraph 2.3 is close to the absolute crystallinity $\chi$. The adjustment proposed in Figure 7 corresponds to $\lambda_{X}=$ 14.8 where $\lambda_{A m}$ values are taken from Figure 5. The agreement is quite satisfying for the traction part and the beginning of recovery. Along the unloading branch, there is a significant discrepancy between the computed and measured crystallinity curves. This indicates a deviation from Eq. (3) along the unloading curve, with the rate of decrease of $\lambda_{\text {am }}$ being comparatively larger than predicted by Eq. (3). This point will be further discussed in Section 3.1.5. Note however that a large part of the discrepancies observed during most of the unloading branch can be attributed to the above-mentioned physical creep: $\lambda_{A m}$ computed from equation (2) is too high and this explains in particular why the computed crystallinity does not return to zero below $\lambda_{\text {Melt }}$. The adjusted $\lambda_{X}=14.8$ value is remarkably close to the estimate $\sqrt{3 N} \approx 12.7$ (with $N$ equal to the average number of Kuhn segments assumed to be equivalent to 1.6 isoprene units; see paragraph 2.1) even though Equation (3) is most probably over-simplified. Note finally that tests based on Flory's original complex formula show a slightly degraded adjustment in particular for the retraction phase.

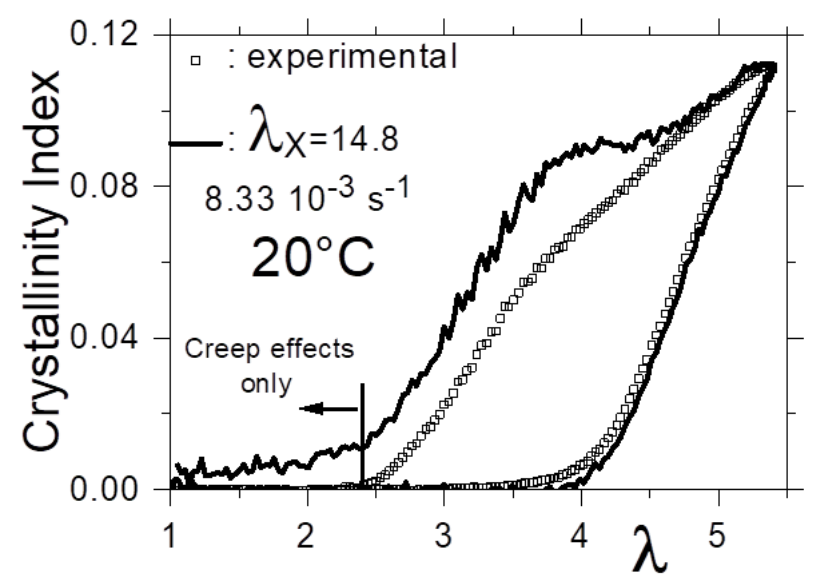

Figure 7. Comparison of the experimental crystallinity index with a calculation based on formula (3) 
As noted above, it also directly follows from Equation (5) together with the condition $d \lambda_{A m} / d t=0$ that the slope $d \chi / d \lambda$ should be independent of the strain rate. This is clearly observed in Figures 3 or 7 above $\lambda_{\text {onset }}$. The effect of strain rate has been investigated over two orders of magnitude in drawing velocity. The maximum strain-rate is essentially limited by the necessity to acquire a sufficient number of diffraction frames. Data obtained at the two extreme drawing velocities are compared in Figures 8-A and B.

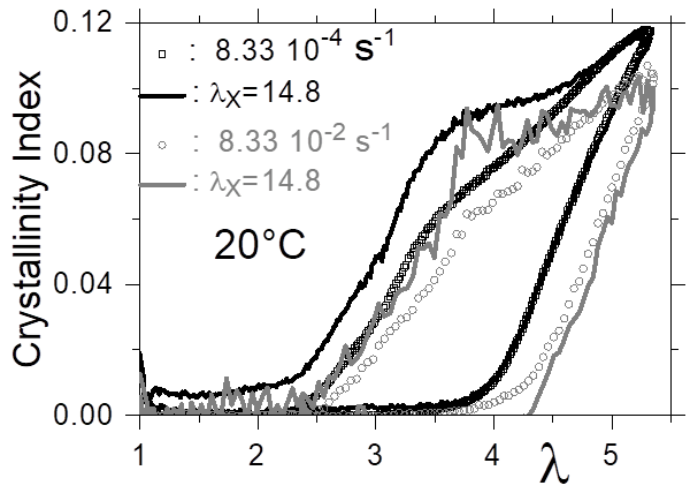

$-\mathrm{A}-$

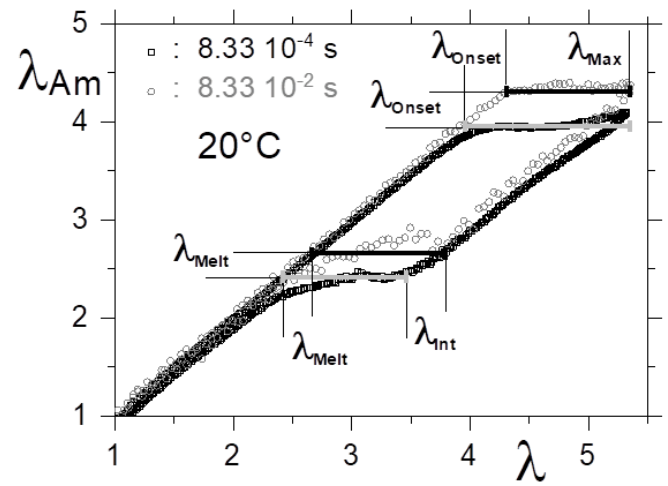

$-\mathrm{B}-$

Figure 8. Dependence of the crystallinity index (A) and local elongation for the amorphous phase (B) upon applied elongation at two strain-rates. Heavy lines (A) are calculations based on formula 2.

The two $\chi$ curves Figure 8-A are very similar in shape, apart from a clear shift of $\lambda_{\text {onset }}$. On the other hand, the slope $d \chi / d \lambda$ for the traction does not show any significant dependence on the imposed strain rate, as reported in Table 2 . Accordingly the maximum $\chi$ value slightly decreases at the higher strain rate. It is again observed that the slope $d \chi / d \lambda$ is independent of the strain rate above $\lambda_{\text {onset }}$, which is a further remarkable manifestation of the strain regulation effect. 
The curves for $\lambda_{A m}$ (Figure 8-B) are also quite similar at both strain rates. In particular the two $\lambda_{\text {Onset }}$ and $\lambda_{\text {Melt }}$ plateaus are well-defined. $\chi$ values computed with Equation (3) are reported in Figure 8-A for $\lambda_{X}=14.7$ (heavy lines). As it was the case in Figure 7, the agreement is quite remarkable for stretching and the beginning of recovery. Characteristic draw ratios are reported in Table 3 for the different strain-rates. $\lambda_{\text {Melt }}$ shows no significant variation, which can be attributed to the absence of melting retardation. The progressive shift to higher values for $\lambda_{\text {Onset }}$ with increasing strain-rate reflects the kinetic aspect of the stretching branch, according to Equation (7) and Figure 6.

\begin{tabular}{|c|c|c|c|}
\hline strain rate $\left(\mathrm{s}^{-1}\right)$ at $20^{\circ} \mathrm{C}$ & $\langle d C / d \lambda\rangle$ & Temperature & $\langle d C / d \lambda\rangle$ \\
\hline $8.3310^{-4}$ & 0.085 & $30^{\circ} \mathrm{C}$ & 0.087 \\
& & & \\
\hline $4.1710^{-3}$ & 0.086 & $\left(8.3310^{-3} \mathrm{~s}^{-1}\right)$ & \\
\hline $8.3310^{-3}$ & 0.085 & & \\
\hline $2.510^{-2}$ & 0.095 & $\left(8.3310^{\circ} \mathrm{C} \mathrm{s}^{-1}\right)$ & \\
& & & \\
\hline $8.3310^{-2}$ & 0.092 & & \\
& & & \\
\hline
\end{tabular}

Table 2. Average slope $<d C I / d \lambda>$ as a function of strain-rate and temperature.

\begin{tabular}{|c|c|c|c|c|}
\hline Initial strain rate $\left(\mathrm{s}^{-1}\right)$ & $\lambda_{\text {Onset }}$ & $\lambda_{\text {Int }}$ & $\lambda_{\text {Int }} *$ & $\lambda_{\text {Melt }}$ \\
\hline $8.3310^{-4}$ & 3.96 & 3.46 & 3.96 & 2.60 \\
\hline $4.1710^{-3}$ & 4.08 & 3.64 & 3.86 & 2.62 \\
\hline $8.3310^{-3}$ & 4.10 & 3.64 & 3.85 & 2.63 \\
\hline $2.510^{-2}$ & 4.25 & 3.82 & 3.79 & 2.72 \\
\hline $8.3310^{-2}$ & 4.31 & 3.79 & 3.65 & 2.64 \\
\hline & & & & \\
\hline
\end{tabular}

Table 3. Dependence of characteristic draw ratios upon strain-rate. 


\subsubsection{Effect of temperature.}

Some measurements were performed at temperatures similar to those used for tensile impact tests and for an intermediate strain rate $\left(8.3310^{-3} \mathrm{~s}^{-1}\right)$. Results are presented in Figure 9-A and $\mathrm{B}$ and the corresponding characteristic draw ratios are collected in Table 4. The adjustment of the crystallinity index by formula (3) is made for $\lambda_{X}$-values slightly higher than at $20^{\circ} \mathrm{C}$ (15.4 and 15.8 to compare with 14.8$)$. In Figure 9-B the plateau at $\lambda_{\text {Onset }}$ remains welldefined, whereas the plateau at melting becomes much less marked.

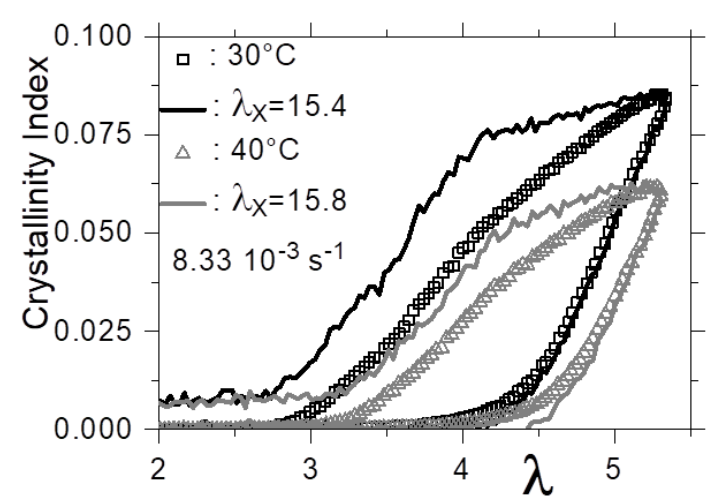

$-A-$

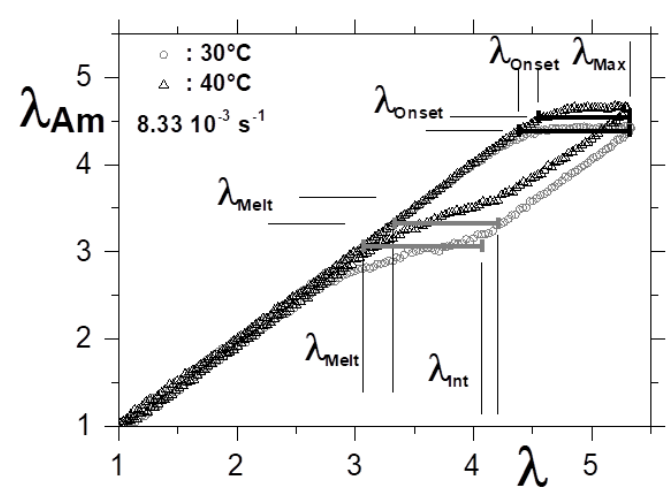

$-\mathrm{B}-$

Figure 9. Dependence of the crystallinity index (A) and local elongation for the amorphous phase (B) upon applied elongation at two temperatures. Heavy lines (A) are calculations based on Equation (3).

\begin{tabular}{|c|c|c|c|c|}
\hline Temperature $\left({ }^{\circ} \mathrm{C}\right)$ & $\lambda_{\text {Onset }}$ & $\lambda_{\text {Int }}$ & $\lambda_{\text {Int }}{ }^{*}$ & $\lambda_{\text {Melt }}$ \\
\hline 20 & 4.10 & 3.64 & 3.85 & 2.63 \\
\hline 30 & 4.39 & 4.10 & 4.00 & 3.07 \\
\hline 40 & 4.55 & 4.21 & 4.10 & 3.33 \\
\hline
\end{tabular}

Table 4. Characteristic draw ratios versus temperature.

\subsubsection{Discussion.}


The above considerations rely on a number of hypotheses that must be discussed. First, SIC kinetics is controlled by the nucleation rate and chain diffusion, and which factor is limiting is still a matter of controversy. In the quiescent state, a maximum in the crystallization rate is observed at ca. $-25^{\circ} \mathrm{C}$ and nucleation is expected to be the limiting factor well-above this temperature. However K.Brüning et al. still favor diffusion-limited kinetics above $-25^{\circ} \mathrm{C}$ for stretched samples ${ }^{17}$. One objection is that a diffusion limited process would result in a steady increase of the crystallite dimensions during crystallization, which is not observed $^{18,19}$. Candau et al. and Gros et al. made a detailed thermodynamic analysis of the parameters governing SIC kinetic and we follow their conclusion that nucleation is the limiting factor well above $25^{\circ} \mathrm{C}^{20,21,22}$. Indeed, it has been stressed by these authors that preextension of amorphous chains lowers the entropy cost for incorporation of chain segments into nuclei, which is in line with the general Flory type argument. Lahgmach et al. and Gros et al. assume that such accumulation can be described by a drastic increase of the effective interfacial energy which enters in the expression of the nucleation barrier ${ }^{22,23}$. The energy barrier for nucleus formation $\Delta H$ thus depends on the macroscopic draw ratio $\lambda$ before SIC onset or on $\lambda_{A m}$ after SIC onset.

The dependence of $\lambda_{\text {Onset }}$ upon drawing velocity is well-documented. In particular, Candau et al. have combined mechanics, infra-red thermometry and X-ray diffraction to measure $\lambda_{\text {onset }}$ over five-decades in strain-rate ${ }^{20}$. Our data are reported in Figure 10 and we observe an approximate logarithmic dependence on the drawing velocity in the explored range, in agreement with previous reports. According to Candau et al., $\Delta H\left(\lambda_{A m}\right)$ may be approximated by a polynomial function of $\lambda_{A m}$ and it can be linearized for small variations in $\lambda_{A m}{ }^{16,21}$. A logarithmic dependence of $\lambda_{\text {onset }}$ on the strain rate $d \lambda / d t$ for a limited range in strain rate is thus expected (see Figure 9). 


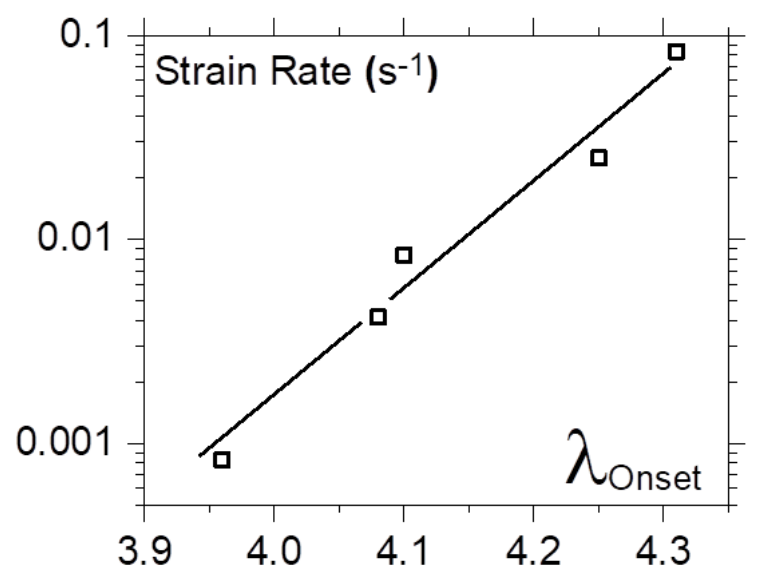

Figure 10. Strain-rate vs elongation at crystallization onset (straight line is a guide for the eye).

During recovery and between $\lambda_{\text {Max }}$ and $\lambda_{\text {Int }}, \lambda_{A m}$ drops almost parallel to the opposite branch $\lambda_{A m}=\lambda$. A possible explanation for this striking observation is as follows: as quoted above, we attribute the stress hardening observed during stretching to extensive crystallite networking. One can thus suppose that the sample is two phases at a microscopic scale with heavily crystallized domains forming the crystallite network and amorphous domains with elongation $\lambda_{A m}$. Note that this two-phase model was also proposed by Fukahori ${ }^{24}$. Recovery is accompanied by a disintegration of the crystallite network (rapid drop of the engineering stress) and independently the amorphous domains relax at the imposed strain-rate. $\lambda_{\text {Int }}$ is the elongation at which the draw ratio for the amorphous domains and for the amorphous fraction of the crystallite network become similar and equal to $\lambda_{\text {Melt }}$. It is to notice that the relation

$$
\lambda_{\text {In }} \approx \lambda_{\text {Max }}+\lambda_{\text {Melt }}-\lambda_{\text {Onset }}
$$

is expected to hold based upon the above-mentioned parallelism of the two branches. At this point, the local two-phase equilibrium ("inverse striction") continues till complete melting and it is reminiscent of the macroscopic two-phase equilibrium observed in weakly crosslinked samples and at moderate temperatures ${ }^{25}$. 
3.1.6. Modelization. We now propose a simple modelization of SIC during cycling elongation that somehow summarizes our results there. An idealized $\lambda_{A m}$ vs $\lambda$ cycle is proposed in Figure 10-A and we use formula (3) to compute the associated $C$ value (Figure 11-B). At a given temperature $\lambda_{X}$ is the only adjustable parameter, whereas $\lambda_{\text {Melt }}$ and $\lambda_{\text {onset }}(d \lambda / d t)$ are experimental parameters. For this last parameter, only two experimental determinations are required if we assume a logarithmic dependence.

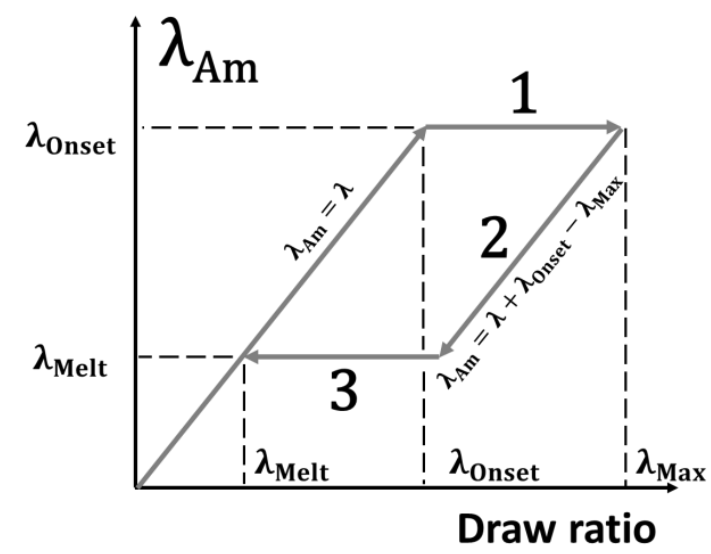

$-A-$

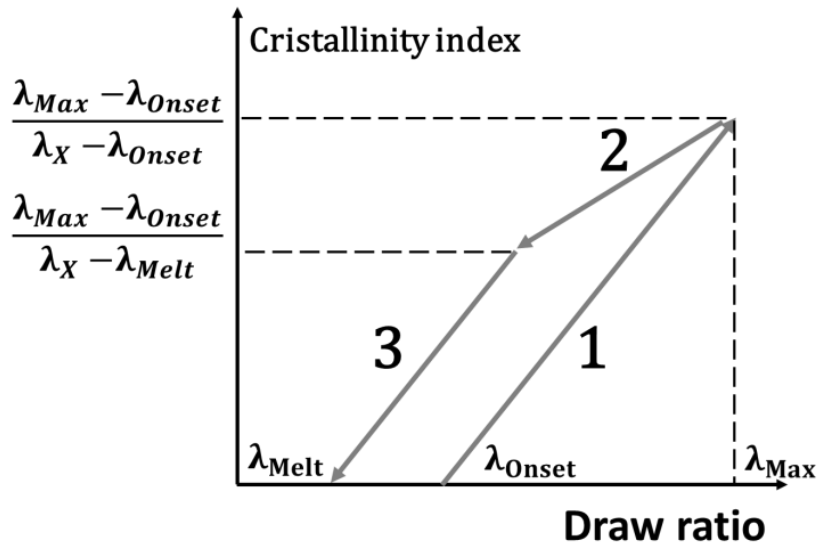

$-B-$

Figure 11. Modelization for the amorphous phase draw ratio (A) and the crystallinity index

(B) during cyclic deformation.

\subsection{Tensile impact tests}

\subsubsection{Experimental configuration.}

We used a symmetric double pull configuration that insures maximum efficiency. In that case, the fixed stroke amplitude provided by the two opposite pneumatic springs is ca. $58 \mathrm{~mm}$. The sample is pre-extended $\mathrm{X} \mathrm{mm}$ prior step-wise extension in order to reach a targeted overall displacement ca. $\mathrm{X}+58 \mathrm{~mm}$. We worked in a range ca. $2-32 \mathrm{~mm}$ for $\mathrm{X}$ values such that no SIC develops prior to step-wise extension and no failure of the sample occurs during 
extension. This corresponds to final, fixed draw ratios in the range $3.70-5.05$, as directly measured by following marks on the samples with an optical camera. Symmetric pull has the drawback that the load sensor is located on a moving part and has to sustain a brutal deceleration at the end of the stretching step: only sensors with capacity inappropriate to our samples can be used and the evolution of the stress cannot be measured accurately. The crystallization rate decreases rapidly with time and the number of recorded frames can be accordingly reduced. An acquisition sequence of 20 frames with 0.4 s exposure time followed by 800 frames with 4 s exposure time was generally used (total experiment duration: ca. $1 \mathrm{hr}$ ).

\subsubsection{Experimental results.}

The time-dependence of the crystallinity index obtained at a temperature of $20^{\circ} \mathrm{C}$ with the acquisition sequence described above is plotted in black symbols in Figure 12-A. At the maximal elongation 5.05, a slope change occurs close to the moment the acquisition time get changed. In order to verify it does not correspond to some artefact, additional data have been collected with an acquisition sequence of 5000 frames with constant $0.4 \mathrm{~s}$ exposure time. They are presented in grey curves and both data sets overlap nearly perfectly. Curves obtained at draw ratios 4.60 and 5.05 are nearly parallel and this is also true for the draw ratio 4.15 for elapsed times higher than ca. 100s. A much lower crystallization rate is observed at an elongation 3.70. It is tantalizing to put these results in perspective with the data collected in Table 2 for $\lambda_{\text {Onset }}$. We see that the lower draw ratio (3.70) is below $\lambda_{\text {Onset }}$ at all investigated strain-rates; elongations 4.60 and 5.05 are always above while elongation 4.15 is intermediate. 


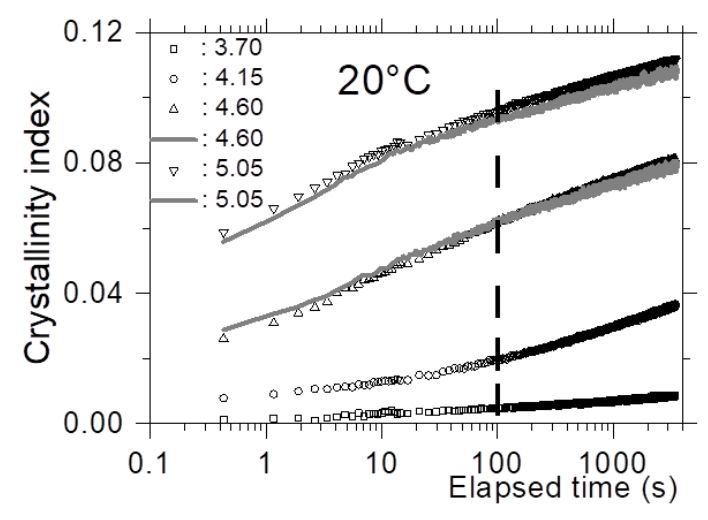

$-\mathrm{A}-$

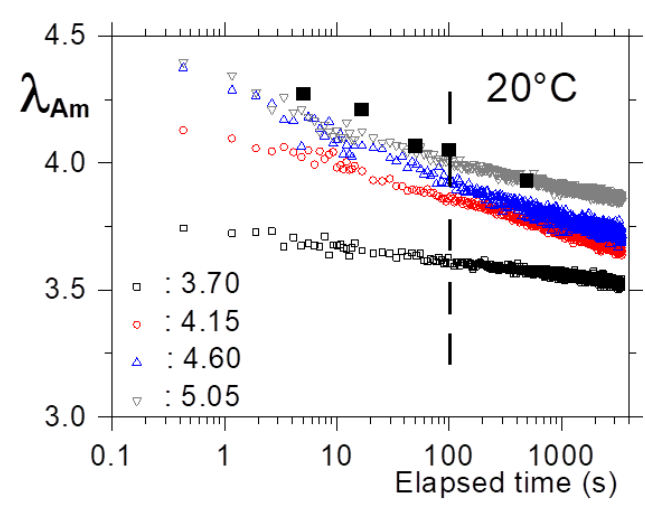

$-\mathrm{B}-$

Figure 12. Time-dependence of the crystallinity index (A) and amorphous phase draw ratio

(B) after step completion $\left(20^{\circ} \mathrm{C}\right)$. (A): grey and black symbols correspond to different acquisition sequences (see text). (B): black squares point to renormalized $\lambda_{\text {Onset }}$.

The time-dependence for the draw ratio of the remaining amorphous fraction, as obtained by applying equation (2), is plotted in Figure 12-B. $\lambda_{A m}$ for the lower draw ratio remains significantly lower and decreases at a much lower rate compared to what is observed for higher draw ratios. Indeed it is striking to see that data for draw-ratios 4.60 and 5.05, and for draw ratio 4.15 above an elapsed time ca. 100s are closely grouped together. In what follows we attempt to compare these grouped values to the $\lambda_{\text {Onset }}$ values measured in mechanical cycling. For this purpose it is necessary to establish some correspondence between strain rate $d \lambda / d t$ during mechanical cycling and elapsed time during tensile impact test. The draw ratio interval that separates the SIC detection limit (where detectable crystallization starts) and $\lambda_{\text {Onset }}$ where $\lambda_{A m}$ starts to remain constant is typically $\Delta \lambda \approx 0.5$ (see Figure 3 ) and the corresponding elapsed time is thus $\Delta \lambda /(d \lambda / d t)$. It is reasonable to take this value as an equivalent elapsed time. The $\lambda_{\text {Onset }}-$ values are plotted in Figure 12-B as black squares as a function of this elapsed time. Keeping in mind the fact that the choice of the elapsed time is 
somehow arbitrary, we see that both sets of data are rather close. Quite convincing is the fact that the $\lambda_{\text {Onset }}-$ values exhibit a time-dependence similar to the tensile impact data.

We conclude this paragraph by a comparison of directly measured $C$ values and values computed using equation (4) (or equivalently (5)). The values reported in Figure 13 have been obtained with a parameter $\lambda_{X}=14.8$ already determined at $20^{\circ} \mathrm{C}$ for cyclic elongation. The agreement is quite satisfying for the two higher draw ratios 4.60 and 5.15. At the end of the measuring time, the computed value is higher by $24 \%$ for $\lambda=4.15$ and almost by $90 \%$ for $\lambda=$ 3.70. These discrepancies are partially explained by the fact that the crystalline content remains low at these draw-ratios and that stress-relaxation, aside from SIC, becomes responsible for a non-negligible fraction of the strain-relaxation. Altogether, Figure 13 shows that the lever rule (equation (3)) remains valid even in out-of-equilibrium situations, as it is essentially a geometrical conditions (or more precisely, a condition of local mechanical equilibrium).

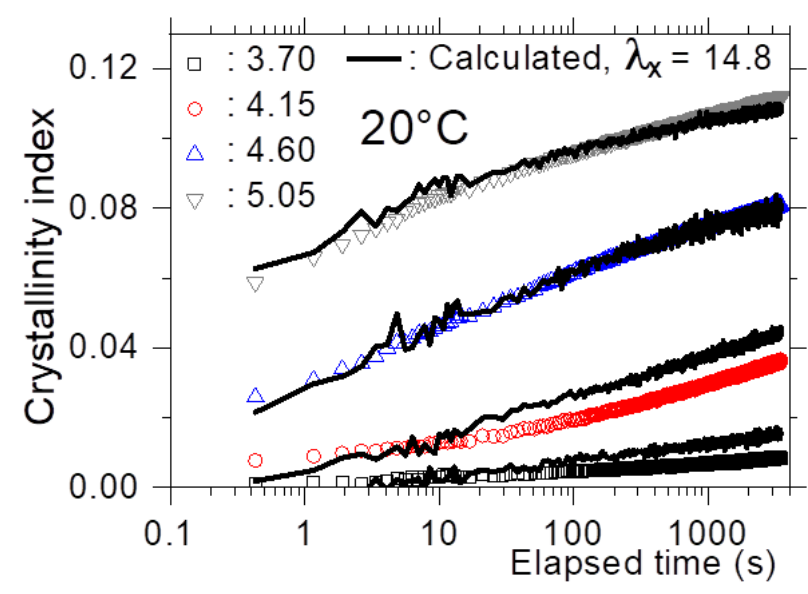

Figure 13. Comparison of experimental and computed crystallinity indices at $20^{\circ} \mathrm{C}$.

Another, equivalent way of demonstrating this fact is to plot the crystallinity index as a function of the amorphous phase draw ratio during each relaxation experiment. The results are 
shown in Figure 14. Plain curves in Figure 14 have been obtained by applying the lever rule (equation (3)) still using the value $\lambda_{X}=14.8$.

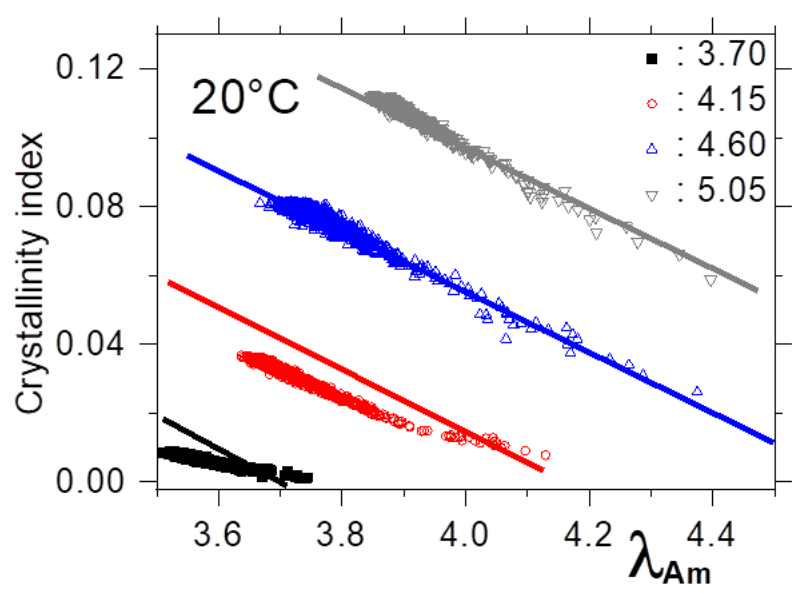

Figure 14. The crystallinity index as a function of the amorphous phase draw ratio. Plain lines are obtained by applying the lever rule (equation (3)).

\subsubsection{Discussion. Time-overstrain superposition.}

The hypothesis that the crystallization rate is controlled only by the nucleation probability that depends upon $\lambda_{A m}$ should in principle enable implementing a heuristic 'time-strain' superposition principle and building a master curve by applying a shift factor to the time scales of the curves obtained at different $\lambda$ values. This is illustrated in Figure 15, in which the master curve corresponding to $\lambda=4.60$ (meaning that the shift factor is one for this draw ratio) is shown. A quite reasonable master curve is indeed obtained in this heuristic way. The corresponding shift factors are reported in Table 5. 


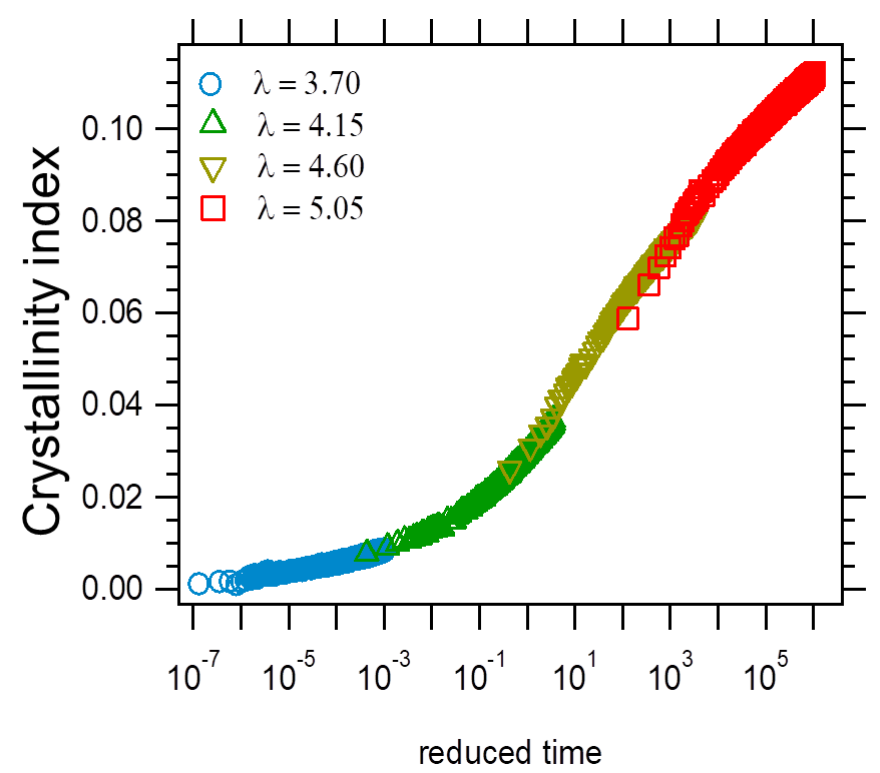

Figure 15. Master curve for the evolution of the crystallinity $\chi$ as a function of time at an equivalent draw ratio $\lambda=4.60$, obtained by applying to the time scale a shift factor $a(\lambda)$ as reported in Table 5.

\begin{tabular}{|c|c|}
\hline$\lambda$ & Shift factor $a(\lambda)$ \\
\hline 3.7 & $3 \times 10^{-7}$ \\
\hline 4.15 & $10^{-3}$ \\
\hline 4.6 & 1 \\
\hline 5.05 & 300 \\
\hline
\end{tabular}

Table 5. The shift factor used to construct the master curve shown in Figures 15 and 16.

Then, according to the hypothesis that the lever rule (equation(3)) remains valid all along the relaxation process, as it was already quite convincingly demonstrated by Figures 13 and 14 , a similar master curve should be obtained for the quantity $\left(\lambda-\lambda_{a m}\right) /\left(\lambda_{c}-\lambda_{a m}\right)$. This is shown in Figure 16. The same shift factors as used in Figure 15 and reported in Table 5 were used. Even though the superposition is not as good as for the crystallinity curves, the overall 
trend is quite illuminating. As noted before, the deviation with respect to curve superposition may be explained by the effect of yield, which comes into play independently from SIC.

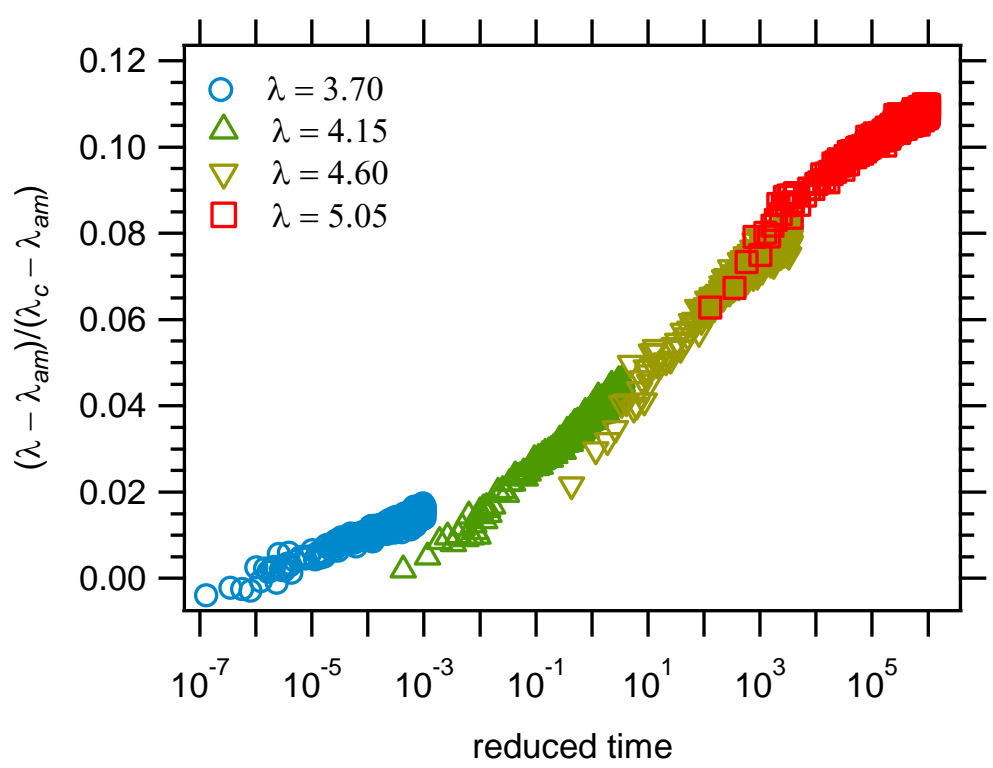

Figure 16. Master curve for the evolution of the quantity $\left(\lambda-\lambda_{a m}\right) /\left(\lambda_{c}-\lambda_{a m}\right)$ as a function of time at an equivalent draw ratio $\lambda=4.60$, obtained by applying to the time scale a shift factor $a(\lambda)$ as reported in Table 5 .

\section{Conclusion.}

The fundamental role played by over-stretching for the SIC kinetic has been already underlined by previous authors: it dramatically increases the crystallization rate, but in turn crystallization relaxes the amorphous fraction and hence diminishes over-stretching. We have shown that the balance between these two contradicting effects leads to the definition of a critical draw ratio for the amorphous fraction that depends upon the time-scale of the measure and that corresponds to the draw ratio at crystallization onset at a macroscopic level. The effect of SIC is to maintain the elongation of the amorphous phase close to this critical value in out-of-equilibrium conditions. A progressive return to close-to-equilibrium as for the recovery part of cyclic deformation results in the rapid drop of the amorphous strain-state to 
levels close to the draw ratio at melting. Based on these considerations, we could introduce a simple model for the time-evolution of the crystalline content during tensile test experiments or cyclic elongation. Most discrepancies compared to the experimental data are observed for the recovery part of cyclic elongation. Indeed we suspect micro-phase separation in that case and a proper description is a first challenge. A second fundamental challenge is a description of stress-hardening during stretching that constitutes one of the most impressive effects of SIC. Physical cross-linking is generally evoked but it should provoke an effect adverse to the observed strain-regulation. Furthermore it cannot explain our observation in NR and CR that similar crystalline contents induce different levels of stress-hardening according to the temperature. We repeatedly propose crystallite networking as the active mechanism, but this proposition still waits to be proven.

\section{References}

[1] Toki, S. The effect of strain-induced crystallization (SIC) on the physical properties of natural rubber, In Manufacture and Applications of Natural Rubber Kohjiya, S., Ikeda, Y., Eds.; WoodHead Publishing, Elsevier 2014.

[2] de Candia, F.; Romano, G.; Russo, R. Stress and thermally induced crystallization in crosslinked cis-polybutadiene. J.Appl. Polym. Sci. 1987, 34, 211-221.

[3] Gent, AN. Crystallization in stretched plymer networks. I. trans-polychloroprene. J.Polym. Sci. Part A 1965, 3, 3787-3801.

[4] Koch, MJH.; Bordas, E.; Schöla, E.; Broecker, HChr. Kinetic study of the crystallization of stretched polyisobutylene using synchrotron radiation. Polym. Bull. 1979, 1, 709-714.

[5] Kojio, K.; Nakamura, S.; Furukawa, M. Effect of side groups of polymer glycol on elongation-induced crystallization behavior of polyurethane elastomers. Polymer 2004, 45, 8147-8152. 
[6] Flory, PJ. Thermodynamics of Crystallization in High Polmers. I. Crystallization Induced by Stretching. J.Chem.Phys. 1947,15, 397-408.

[7] Plagge, J.; Klüpple, M. A theory relating crystal size, mechanical response, and degree of crystallization in strained natural rubber. Macromolecules 2018, 51, 3711-3721.

[8] Huneau, B. Strain-induced crystallization of natural rubber: a review of X-ray diffraction investigations. Rubber Chem Technol. 2011, 84, 425-452.

[9] Albouy, P-A.; Vieyres, A.; Pérez-Aparicio, R.; Sanséau, O.; Sotta, P. The impact of straininduced crystallization on strain during mechanical cycling of cross-linked natural rubber. Polymer 2014, 55, 4022-4031.

[10] Le Gac, P-Y.; Albouy, P-A.; Petermann, D. Strain-induced crystallization in an unfilled polychloroprene rubber: kinetics and mechanical cycling. Polymer 2018, 14, 209-217.

[11] Tosaka, M.; Senoo, K.; Sato, K.; Noda, M.; Ohta, N. Detection of fast and slow crystallization processes in instantaneously-strained samples of cis-1.4-polyisoprene. Polymer 2012, 53, 864-872.

[12] K Brüning, K.; Schneider, K.; Roth, SV.; Heinrich, G. Kinetics of Strain-Induced Crystallization in Natural Rubber Studied by WAXD: Dynamic and Impact Tensile Experiments. Macromolecules 2012, 45, 7914-7919.

[13] Valentín, JL.; Psadas, P.; Fernández-Torres, A.; Malmierca, MA.; Gonzáles, L.; Chassé, W.; Saalwächter, K. Inhomogeneities and Chain Dynamics in Diene Rubbers Vulcanized with Different Cure Systems. Macromolecules 2010, 43, 4210-4222.

[14] Nobbs, JH.; Bower, DI. Orientation averages for drawn rubber networks. Polymer 1978, 19, 1100-1103. 
[15] Vieyres, A.; Pérez-Aparicio, R.; Albouy, P-A.; Sanséau, O.; Saalwächter, K.; Long, DR.; Sotta, P. Sulfur-Cured Natural Rubber Elastomer Networks: Correlating Cross-Link Density, Chain Orientation, and Mechanical Response by Combined Techniques. Macromolecules 2013, 46, 889-899.

[16] Albouy, P-A.; Sotta, P. Strain-Induced Crystallization in Natural Rubber, In Polymer crystallization II: from chain microstructure to processing; Advances in Polymer Science; Springer, 2017, 167-205

[17] Brüning, K.; Schneider, K.; Roth, SV.; Heinrich, G. Kinetics of strain-induced crystallization in natural rubber: a diffusion-controlled rate law. Polymer 2015, 72, 52-58.

[18] Brüning, K.; Schneider, K.; Roth, SV.; Heinrich, G. Kinetics of strain-induced crystallization in natural rubber studied by WAXD: dynamic and impact tensile experiments. Macromolecules 2012, 45, 7914-7919.

[19] Tosaka, M.; Senoo, K.; Sato, K.; Noda, M.; Ohta, N. Detection of fast and slow crystallization processes in instantaneously-strained samples of cis-1.4-polyisoprene. Polymer 2012, 53, 864-872.

[20] Candau, N.; R Laghmach, R.; Chazeau, L.; Chenal, J-M.; Gauthier, C.; Biben, T.; Munch, E. Influence of strain rate and temperature on the onset of strain induced crystallization in natural rubber. Eur. Polym. J. 2015, 64, 244-252.

[21] Candau, N.; Laghmach, R.; Chazeau, L.; Chenal, J-M.; Gauthier, C.; Biben, T.; Munch, E. Strain-induced crystallization of natural rubber and crosslink densities heterogeneities. Macromolecules 2014, 47, 5815-5824.

[22] Gros, A.; Tosaka, M.; Huneau, B.; Verron, E.; Poomprabud, S.; Senoo, K. Dominating factor of strain-induced crystallization in natural rubber. Polymer 2015, 76, 230-236. 
[23] Laghmach, R.; Candau, N.; Chazeau, L.; Munch, E.; Biben, T. Phase field modelling of strain induced crystal growth in an elastic matrix. J. Chem. Phys. 2014, 142, 244905.

[24] Fukahori, Y. Mechanism of the self-reinforcement of cross-linked NR generated through the strain-induced crystallization, Polymer 2010, 51 1621-1631.

[25] Albouy, P-A.; Marchal, J.; Rault, J. Chain orientation in natural rubber, Part I: The inverse yielding effect. Eur. Phys. J. 2006, E17, 247-259. 
$\underline{\text { For Table of Contents use only }}$

DRAW RATIO AT ONSET OF STRAIN-INDUCED CRYSTALLIZATION IN CROSSLINKED NATURAL RUBBER.

Pierre-Antoine Albouy and Paul Sotta
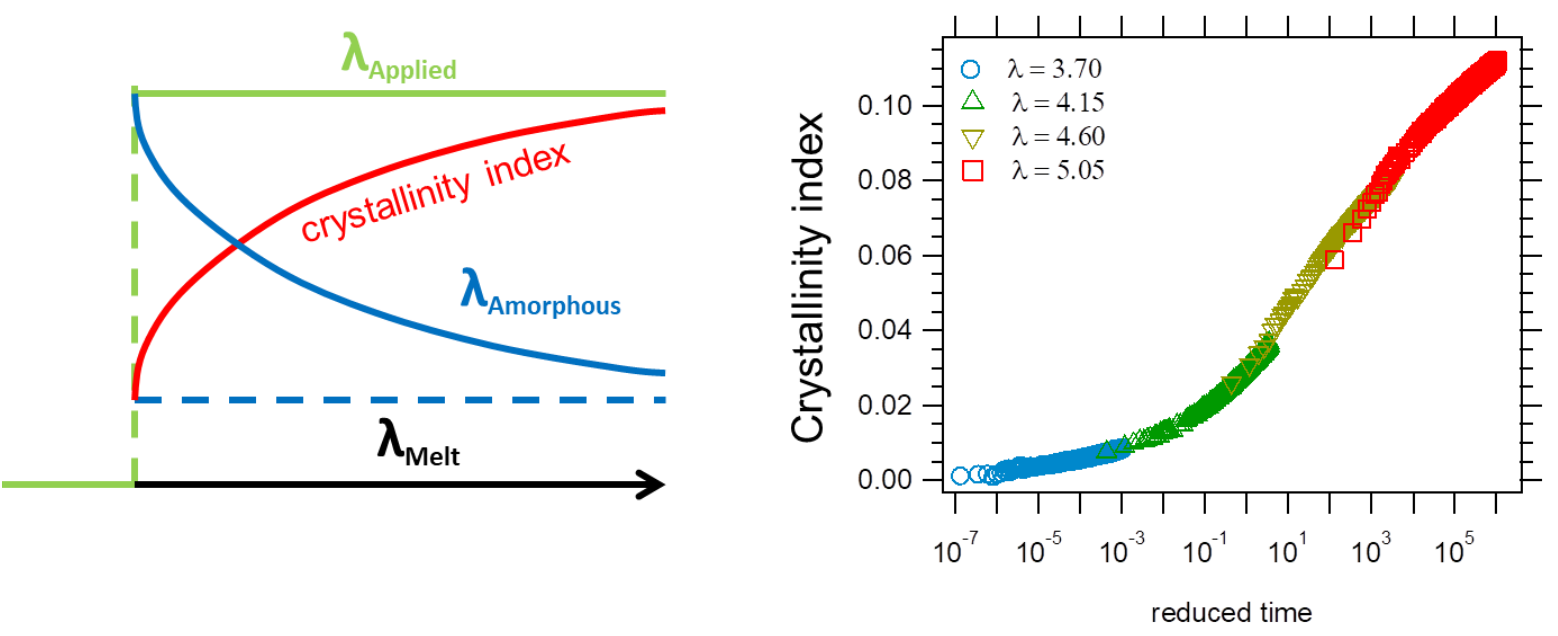